\title{
Acute bilateral paramedian thalamic and mesencephalic infarcts due to occlusion of the Percheron artery: a case report
}

\author{
Alev Leventoğlu $\odot$, Gülin Morkavuk $\odot$ \\ Department of Neurology, Ufuk University School of Medicine, Ankara, Turkey
}

DOI: $10.18621 /$ eurj.447002

\begin{abstract}
The thalamus and midbrain mesencephalon have a complex blood supply with a great number of feeding arteries. The blood build up of thalamus is being provided by four arteries originating from the vertebrobasilar system and posterior communicating artery. The paramedian thalamic artery is derived from the posterior cerebral artery proximal P1 segment. The infarcts of arterial constitute approximately $35 \%$ of all thalamic infarcts. However, bilateral infarctions of this region are very infrequent. Percheron artery originates from the first branch of the posterior cerebral artery and gives bilateral medial thalamic perforating branches. We described a patient of a 88-year-old woman with acute bilateral thalamic and midbrain infarcts due to occlusion of Percheron artery demonstrated on magnetic resonance imaging.
\end{abstract}

Keywords: Percheron artery occlusion, thalamic infarct, mesencephalic infarct, magnetic resonance imaging

Received: June 23, 2018; Accepted: April 30, 2019; Published Online: July 27, 2019

$\mathrm{T}$ he thalamus and midbrain mesencephalon have a complex blood supply with a great number of feeding arteries [1]. The blood build up of thalamus is being provided by four arteries originating from the vertebrobasilar system and posterior communicating artery. From these, the paramedian thalamic artery (thalamoperforan) also feeds the medial region of the upper brain stem. The paramedian thalamic artery is derived from the posterior cerebral artery proximal P1 segment. The infarcts of arterial constitute approximately $35 \%$ of all thalamic infarcts [2]. However, bilateral infarctions of this region are very infrequent. The proposed mechanism in bilateral thalamic infarction; is a single paramedian thalamic artery occlusion without central dissociation.

The artery of Percheron (AOP) is named after the frenchman, Gerard Percheron, who described it in 1973. This artery originates from the first branch of the posterior cerebral artery and gives bilateral medial thalamic perforating branches. The occlusion was frequently aetiology of cardiac embolism occuring as a result of atrial fibrillation [3]. According such studies, AOP infarct acoount for $\% 0.1$ and $\% 0.3$ of all ischemic strokes and 22 to $35 \%$ of all thalamic infarcts [4]. An ischemic stroke in the territory of an artery of Percheron usually present with several symtoms, which are found in patients with bilateral paramedian thalamic and mesencephalic infarct. These are; memory impairment, vertical gaze palsy, and coma [1].

We described a patient of an 88-year-old woman with acute bilateral thalamic and midbrain infarcts due to occlusion of AOP demonstrated on magnetic resonance imaging.

\section{CASE PRESENTATION}

An 88-year-old woman with a past history of

Address for correspondence: Gülin Morkavuk, MD., Assistant Professor, Ufuk University School of Medicine, Department of Neurology, Ankara, Turkey E-mail:drgcmor@yahoo.com,Tel: +903122044232 
hypertension, ischemic cardiopathy and atrial fibrillation was found unconscious in the early morning by her relatives. She was treated with longterm oral anticoagulants (warfarine), but with poor therapeutic range. On admission, his heart rate was 110/minute and regularly, blood pressure was 140/90 $\mathrm{mmHg}$, respiratory rate was $15 /$ minute and axillary temperature was $36.2{ }^{\circ} \mathrm{C}$.

On neurogical examination, her Glasgow Coma Scale (GCS) score was 6/15 (E1M4V1); small but reactive pupils of the same size, absent oculocephalic reflexes, and Babinski's sign bilaterally. There was no vertical gaze palsy. At presentation, patient had minimal motor response to painful stimuli on the right arm and leg. These findings suggested a brainstem infarction. Complete blood picture, routine biochemistry, throid function tests, vitamin B12 and folate were all within normal limits.

The initial computed tomography (CT) showed no acute hemorrhage or brain lesion. ECG showed atrial fibrillation. Transthoracic echocardiography showed grade 2 diastolic dysfunction. The electroencephalogram (EEG) showed diffuse slowing teta-delta waves.
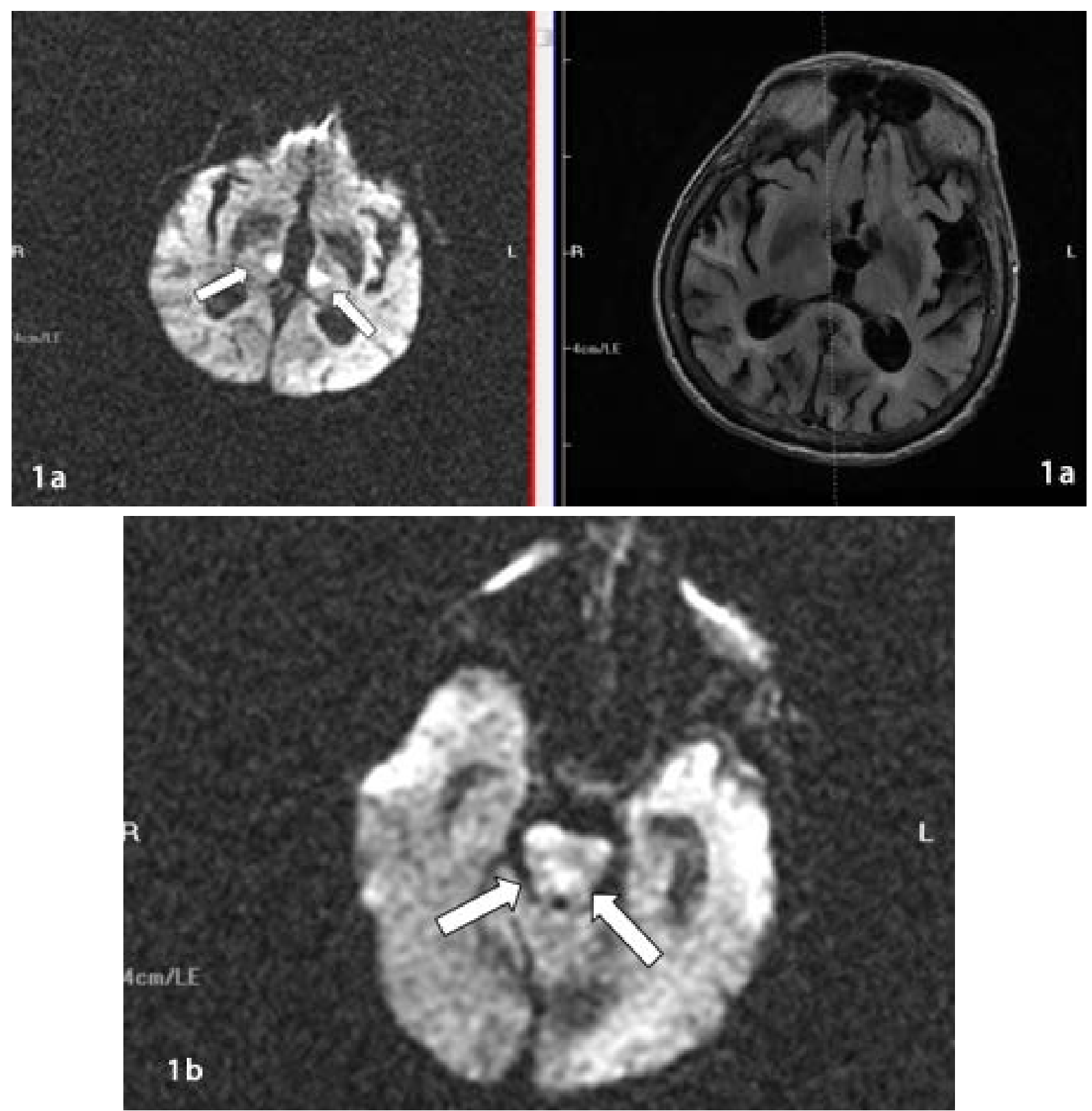

Figure 1. Magnetic resonance imaging shows high signal intensity areas on T2 weighted, FLAIR and diffusion sequences bilaterally and symmetrically infarcts in the medial thalamic region and rostral mid-brain region, right anterior, posterosuperior cerebellar hemisphere and left occipital lobe. (1a and 1b). 


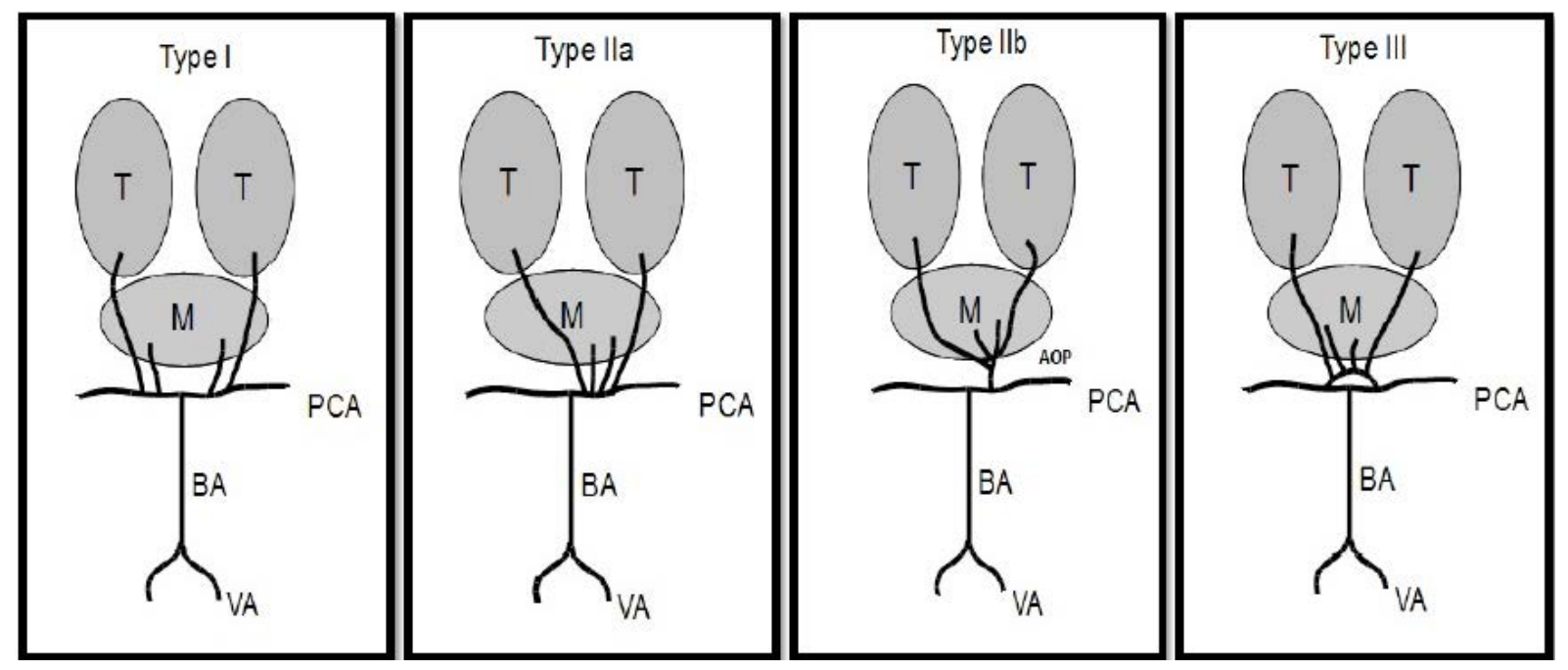

Figure 2. According to Percheron; there are four types of vascular anatomy of the thalamus and mesencephalon. (Type I) Each perforating artery occurs from each right and left posterior cerebral artery, (Type IIa) Perforating arteries occurs directly proximal segment of one of posterior cerebral arteries, (Type IIb) The bilateral perforating arteries arise from one central artery called the artery of Percheron, which arises from the P1 segment of one posterior cerebral artery., and (Type III) An arcade of perforating branches arising from an artery bridging the P1 segments of both posterior cerebral arteries.

Magnetic resonance imaging (MRI) showed high signal intensity areas on T2 weighted, FLAIR and diffusion sequences bilaterally and symmetrically infarcts in the medial thalamic region and rostral midbrain region, right anterior, posterosuperior cerebellar hemisphere and left occipital lobe (Figure 1a and 1b). MR angiography did not reveal any significant finding.

Low-molecular-weight heparin, mannitol, aspirin, atorvastatin, metoprolol in standard recommended dosages was started to patient. At sixth day she became pulmonary infection. She was treated with antibiotics. During her hospitalization, at the $30^{\text {th }}$ day of coma, the patient has passed away due to sysmetic complications.

\section{DISCUSSION}

The main thalamic blood supply comes from the posterior communicating arteries and the perimesencephalic segment of the PCA. Although there is a significant variation and overlap, thalamic vascular territories: inferolateral, anterior, paramedian and posterior. The paramedian thalamic arteries have great variability with respect to number, size and territorial contribution.

According to Percheron; there are four types of vascular anatomy of the thalamus and mesencephalon (Figure 2). Type I is most common. Each perforating artery occurs from each right and left posterior cerebral artery. Type IIa is asymmetrical variant. Perforating arteries occurs directly proximal segment of one of posterior cerebral arteries. Type IIb: The bilateral perforating arteries arise from one central artery called the artery of Percheron, which arises from the P1 segment of one posterior cerebral artery. Type III is an arcade of perforating branches arising from an artery bridging the P1 segments of both posterior cerebral arteries.

In the AOP infarcts, as a result of anatomical variations of paramedian arteries, ischemic patterns are being developed. Bilateral paramedian and / or polar thalamic infarcts as well as bilateral thalamic infarcts and mesencephalon infarcts can be observed [4]. In a study conducted, four types of ischemic patterns were identified that matched the AOP feeding area. The most common is bilateral paramedian thalamic infarct and mesencephalon infarction association, and is observed in $43 \%$. Types of paramedian thalamic infarcts without a mesencephalon infarction of $38 \%, 14 \%$ were accompanied by anterior thalamus and mesencephalon involvement, and presence of bilateral paramedian infarct and $5 \%$ of the cases in which the mesencephalon was preserved and the anterior 
thalamus was retained, were being described [4]. When we look at the ischemic pattern of our case, it is observed that it is the most frequent type.

The most frequent cause of arterial occlusion in the pathogenesis is small vascular disease. In these cases where thromboembolic processes are occured in the second most frequent, it has been reported that the embolic source is mostly the heart, vertebrobasilar system and aorta. It is known that the greatest cause of bilateral thalamic infarcts is cardioembolism [5]. Another possible causes of bilateral thalamic lesions may also due to infections, infiltrative neoplasm, venous occlusison and inflammatory lesions [6].

Infarcts in midbrain and/or bilateral paramedian thalamic region may result in various clinical symptoms; altered mental status, memory impairment $(58 \%)$, vertical gaze palsy $(65 \%)$, motor deficits, confusion (53\%) and coma (42\%) [6]. Altered mental status may present ranging from drowsiness or confusion to hypersomnolance or coma. In our patient presented with coma and persisted until death.

Early diagnosis in percheron arterial infarcts is important in terms of thrombolytic therapy. Kostanian et al. [7], reported that they had a positive result in a patient with percheronian artery occlusion with intraarterial thrombolysis.

\section{CONCLUSION}

Percheron arterial occlusion should be considered if no pathology is detected in the basilar artery in patients admitted to clinic with basilar peak syndrome.

\section{Informed consent}

Written informed consent was obtained from the patient for publication of this case report and any accompanying images.

\section{Conflict of interest}

The authors declared that there are no potential conflicts of interest with respect to the research, authorship, and/or publication of this article.

\section{Acknowledgement}

This case report was presented as a poster in 1EFNS-ENS Congress, Istanbul, Turkey, 2014.

\section{REFERENCES}

[1] Lamot U, Ribaric I, Popovic KS. Artery of percheron infarction: review of literature with a case report. Radiol Oncol 2015;49:141-6.

[2] Tilki HE, Er D, İncesu L. [Acute aphasia after percheron artery occlusion]. SDÜ Sağllk Bilimleri Esntitüsü Dergisi 2011;2:39-44. [Article in Turkish]

[3] Lopez-Serna R, Gonzalez-Carmona P, Lopez-Martinez M. Bilateral thalamic stroke due to occlusion of artery of percheron in patient with patent foramen ovale: a case report. J Med Case Reports 2009,3:7392.

[4] Lazzaro NA, Wright B, Castillo M, Fischbein NJ, Glastonbury CM, Hildenbrand PG, et al. Artery of percheron infarction: imaging patterns and clinical spectrum. AJNR Am J Neuroradiol 2010;31:1283-9.

[5] Yamamoto Y, Georgiadis AL, Chang HM, Caplan LR. Posterior cerebral artery territory infarcts in New England Medical Center Posterior Circulation Registry. Arch Neurol 1999;56:824-32.

[6] Kumral E, Evyapan D, Balkir K, Kutluhan S. Bilateral thalamic infarction. Clinical, etiological and MRI correlates. Acta Neurol Scand 2001;103:35-42.

[7] Kostanian V, Cramer SC. Artery of Percheron trombolysis. AJNR Am J Neuroradiol 2007;28:870-1. 